Check for updates

Cite this: RSC Adv., 2021, 11, 35937

Received 28th August 2021

Accepted 27th October 2021

DOI: $10.1039 / \mathrm{d} 1 \mathrm{ra0} 6503 \mathrm{C}$

rsc.li/rsc-advances

\section{Does electrophilic activation of nitroalkanes in polyphosphoric acid involve formation of nitrile oxides? $\uparrow$}

\author{
Alexander V. Aksenov, (D) ${ }^{* a}$ Nicolai A. Aksenov, (D) ${ }^{a}$ Nikita K. Kirilov, ${ }^{a}$ \\ Anton A. Skomorokhov, ${ }^{a}$ Dmitrii A. Aksenov, (D) a Igor A. Kurenkov, (iD a \\ Elena A. Sorokina, (D) ${ }^{b}$ Mezvah A. Nobic and Michael Rubin (D)*ac
}

The mechanistic rationale involving activation of nitroalkanes towards interaction with nucleophilic reagents in the presence of polyphosphoric acid (PPA) was re-evaluated. Could nitrile oxide moieties be formed during this process? This experiment demonstrates that at least in some cases this could happen, as generated nitrile oxides were successfully intercepted as adducts of [3+2] cycloadditions.

\section{Introduction}

Nitroalkanes possessing acidic $\alpha-\mathrm{CH}$ bonds are traditionally employed as versatile nucleophilic components in a variety of base-assisted $\mathrm{C}-\mathrm{C}$ bond-forming reactions. ${ }^{1-8}$ Another mode of activation of nitroalkanes enables acid-promoted tautomerization into nitronic acid (aci form), which is electrophilic in nature and can interact with a number of nucleophiles. In aqueous media, facile acid-assisted reaction with water takes place, commonly known as the Nef reaction, ${ }^{\mathbf{9 - 1 2}}$ resulting in the formation of aldehydes. In anhydrous media, alternative reactivity patterns are possible, involving interaction with various Cand N-based nucleophiles. Over the last decade, our group has been instrumental in the development of multistep cascade transformations triggered by initial electrophilic activation of nitroalkanes in the presence of polyphosphoric acid (PPA). ${ }^{\mathbf{1 3 - 1 7}}$ Proposed mechanistic rationales for all these processes involve formation of a phosphorylated nitronate species as a key electrophilic intermediate. Herein, we wish to disclose our recent studies proving that formation of nitrile oxide species is also possible under the same conditions. This suggests that alternative mechanisms could be potentially considered, at least for some of the earlier described processes.

${ }^{a}$ Department of Chemistry, North Caucasus Federal University, 1 a Pushkin St., Stavropol 355017, Russia. E-mail: aaksenov@ncfu.ru

'Organic Chemistry Department, Peoples' Friendship University of Russia (RUDN University), 6, Miklukho-Maklaya St., Moscow, 117198, Russian Federation

'Department of Chemistry, University of Kansas, 1567 Irving Hill Road, Lawrence, KS 66045, USA.E-mail: mrubin@ku.edu

$\dagger$ Electronic supplementary information (ESI) available: Spectral charts. CCDC 2099762. For ESI and crystallographic data in CIF or other electronic format see DOI: $10.1039 / \mathrm{d} 1 \mathrm{ra} 06503 \mathrm{c}$

\section{Results and discussion}

As discussed above, it was previously stated that interaction of nitroalkane 1 with PPA leads to the formation of electrophilic nitronate species 2 , enabling subsequent reactions with various nucleophiles. The resulting phosphorylated $N$-hydroxy- $N$-alkyl hydroxylamine 4 may undergo further elimination of the orthophosphoric acid molecule to afford species 5 (oximes or amidines resulting from reaction with carbon or nitrogen-based nucleophiles, respectively, Scheme 1). Oximes $5(\mathrm{Nu}=\mathrm{Ar})$, as

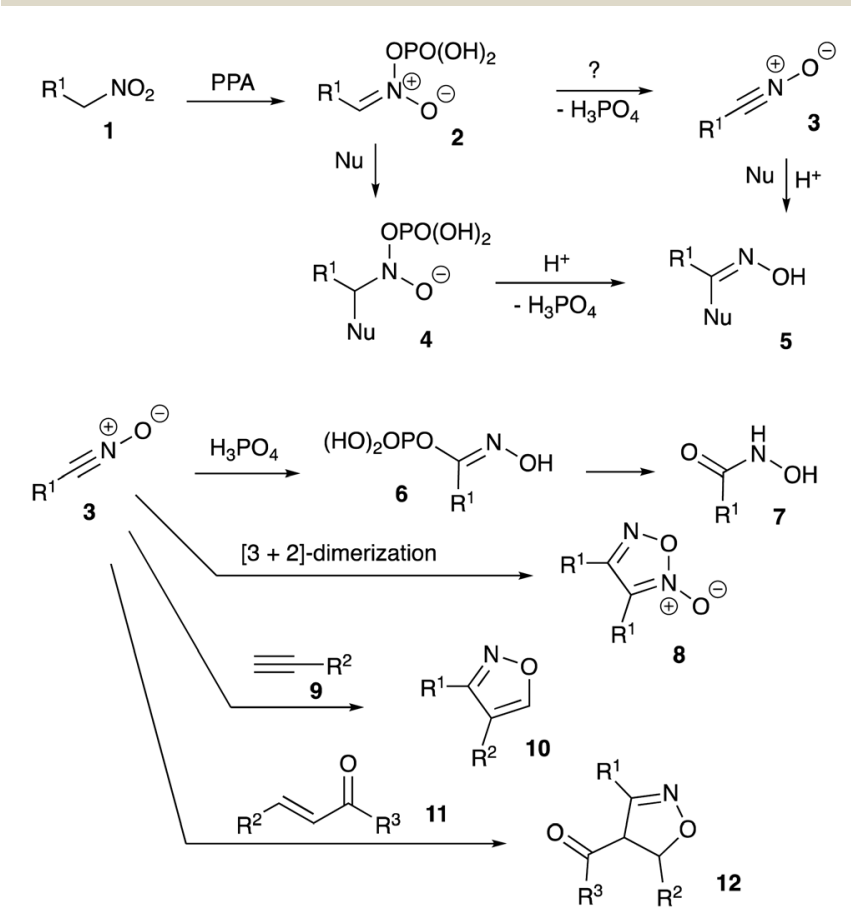

Scheme 1 
Table 1 Optimization of the reaction conditions for generation of nitrile oxide $3 a$ and its subsequent dimerization into furoxan $8 a$

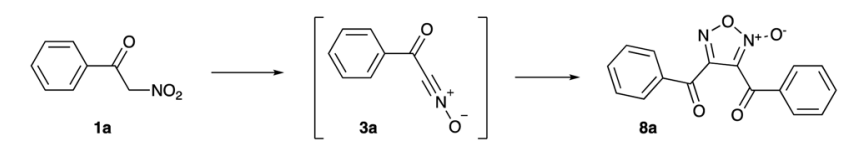

\begin{tabular}{lllll}
\hline & medium & $\begin{array}{l}\text { m (PPA) per mmol } \\
\text { of } \mathbf{1 a}(\mathrm{g})\end{array}$ & $\begin{array}{l}\text { T, }{ }^{\circ} \mathrm{C} \\
(\text { time, h) }\end{array}$ & Yield $^{a}, \%$ \\
\hline 1 & PPA, 80\% & 1.5 & $50(2)$ & 23 \\
2 & & 2 & $50(2)$ & 30 \\
3 & PPA, 87\% & 2 & $50(2)$ & 33 \\
4 & & 2 & $\mathbf{7 0}(\mathbf{0 . 5})$ & $\mathbf{5 1}$ \\
5 & 2 & $90(0.3)$ & 25 \\
${ }^{a}$ & & & \\
\end{tabular}

was previously demonstrated, may participate in Beckmanntype rearrangements, which was employed in acetamidation and carboxyamination reactions. ${ }^{18,19}$ Generation of amidines 5 $(\mathrm{Nu}=\mathrm{NHR})$ were utilized in design of cascade transformations leading to various heterocyclic scaffolds. ${ }^{13,20,21}$ We pondered if elimination of $\mathrm{H}_{3} \mathrm{PO}_{4}$ entity can take place in species 2 to form nitrile oxide 3 under the described reaction conditions. ${ }^{22}$ This species is also inherently electrophilic and reactions with nucleophiles should provide the same type of product $\mathbf{5}$ (Scheme 1).

Furthermore, reaction of 3 with ortho-phosphoric acid in the absence of other nucleophiles could be envisioned, producing $\mathrm{N}$-hydroxyalkanimidic phosphoric anhydride 6, which after hydrolytic cleavage, should provide hydroxamic acid 7. It should be noted that the isomerization of nitroalkanes into hydroxamic acids taking place in the presence of PPA is well documented. ${ }^{23}$ On the other hand, in most typical reactions nitrile oxides act as 1,3-dipoles. If nitrile oxides are indeed generated from nitroalkanes in the presence of PPA, then in the absence of external dipolarophiles, they should undergo $[3+2]$-dimerization to provide 1,2,5-oxadiazole 2-oxides (furoxans) 8 (Scheme 1). Alternatively, cross-cycloaddition reactions with alkynes $\mathbf{9}$ and chalcones $\mathbf{1 1}$ could be performed to generate isoxazoles $\mathbf{1 0}$ or 4,5-dihydroisoxazoles 12, respectively (Scheme 1).

First, we attempted to conduct the experiment involving interaction of 1-nitropropane $\left(\mathbf{1}, \mathrm{R}^{1}=\mathrm{Et}\right.$, Scheme 1$)$ with PPA in the absence of any other reagents by targeting detection for furoxan $8\left(\mathrm{R}^{1}=\mathrm{Et}\right)$ resulting from $[3+2]$-homodimerization. However, formation of such product was not detected, as almost the entire amount of starting nitroalkane was transformed into $N$-hydroxypropionamide $7\left(\mathrm{R}^{1}=\mathrm{Et}\right) \cdot{ }^{24}$ Evidently, even if formed under the described reaction conditions, propionitrile oxide $\left(3, \mathrm{R}^{1}=\mathrm{Et}\right)$ is much too reactive and quickly interacts with the medium. To address this issue, we decided to test the same idea by employing 2-nitro-1-phenylethan-1-one (1a), producing greater concentration of aci-form, which should translate in higher concentration of benzoyl cyanide $N$ oxide (3a). Therefore, this starting material should be more prompt towards the desired dimerization reaction.

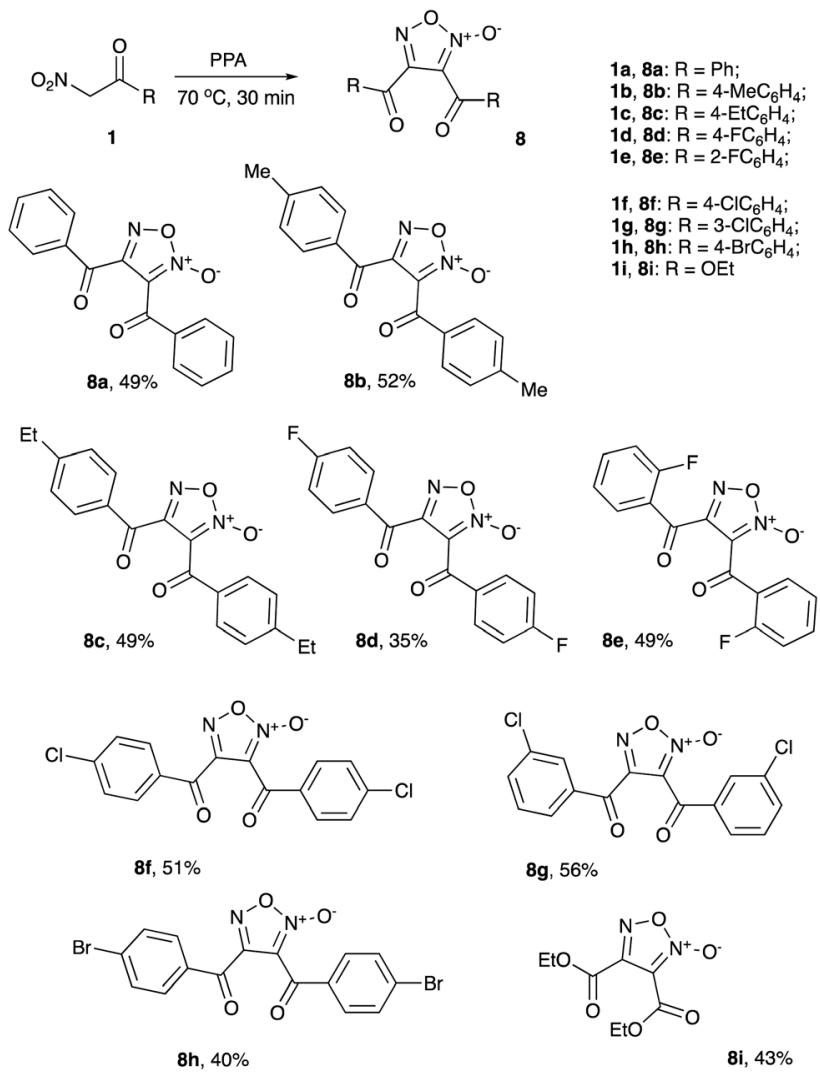

Scheme 2

Initial tests were carried out in PPA with $80 \%$ content of $\mathrm{P}_{2} \mathrm{O}_{5}$ (corresponding to diphosphoric acid, $\mathrm{H}_{4} \mathrm{P}_{2} \mathrm{O}_{7}$ ). A mixture of 1a $(1 \mathrm{mmol})$ with PPA $(1.5 \mathrm{~g})$ was stirred and heated at $50{ }^{\circ} \mathrm{C}$ for 2 hours, stopping only after TLC analysis proved complete consumption of starting material. Delightfully, some nitroalkane was indeed converted into furoxan 8a, albeit in marginal yield (Table 1, entry 1). Increasing the amount of PPA $(2.0 \mathrm{~g})$ improved the yield, though not significantly (entry 2). We thought that switching to $87 \%$ PPA might enhance the reaction performance, since this medium has less nominal content of water and thus less nucleophilic; however, the reaction proceeded sluggishly and at $50^{\circ} \mathrm{C}$, the yield of 8 a was essentially the same as in $80 \%$ PPA (entry 3). Further improvement was achieved upon heating to a higher temperature, which also allowed to cut down reaction time. At $70{ }^{\circ} \mathrm{C}$, a maximum yield of $51 \%$ was obtained (entry 4) and further increases of temperature proved detrimental (entry 5). The purified product 8a was isolated in a $46 \%$ yield (Scheme 2). With optimized reaction conditions in hand, we moved towards scope and limitation studies, the results of which are shown in Scheme 2. Generally, the reactions of substituted 2-nitro-1-phenylethan-1-ones $\mathbf{1 b}$-h proceeded smoothly, well tolerating alkyl and aromatic halide substituents and affording the corresponding furoxans in moderate yields. We also managed to execute successful transformations of ethyl 2-nitroacetate (1i) into furoxan $\mathbf{8 i}$, bearing two ester functions under the same reaction conditions (Scheme 2). 


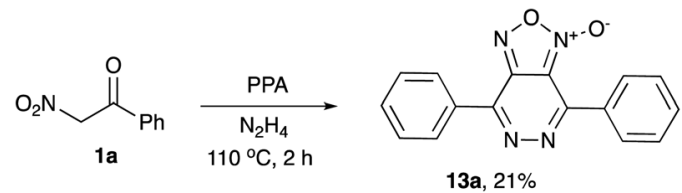

Scheme 3

The possibility for a one-pot assembly of a bicyclic structural core, including a furoxan moiety fused to pyridazine ring, was also evaluated. To this end, nitroketone 1a was treated with PPA in the presence of hydrazine hydrate. The bicyclic structure 13a was formed as a sole isolable product, although in low quantities (Scheme 3).

Next, we decided to explore the possibility to intercept the nitrile oxides generated from nitroalkanes with external dipolarophiles, such as terminal acetylenes. Treatment of various nitroalkanes with PPA was carried out in the presence of phenyl acetylene (9a), 1-heptene (9b), or propargyl bromide (9c) to yield the desired isoxazoles 10 (Scheme 4). Formation of isoxazole 10da was unambiguously confirmed by single crystal X-ray crystallography (Fig. 1).

This reaction cannot be recommended as a preparative protocol towards these materials, since most amount of nitroalkanes were converted into the corresponding hydroxamic acids. Despite that, it clearly confirms that nitrile oxides were indeed generated under the described reaction conditions.

Interception of nitrile oxides with alkenes were tested as well. Reaction of 1a in the presence of chalcone 11a gave nearly

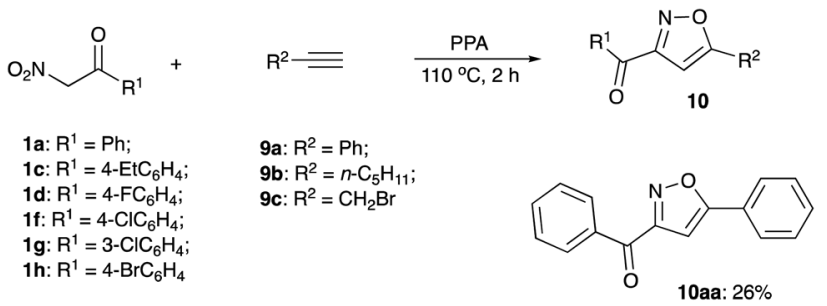

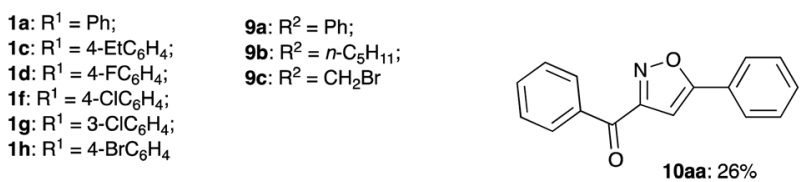

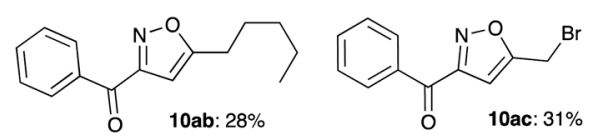

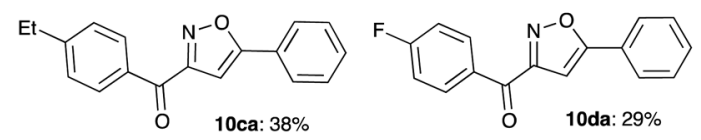<smiles>O=C(c1ccc(Cl)cc1)c1cc(-c2ccccc2)on1</smiles><smiles>CCCCCc1cc(C(=O)c2ccc(Cl)cc2)no1</smiles><smiles>CC(C)(C)O[Na]</smiles><smiles>O=C(c1ccc(Br)cc1)c1cc(CBr)on1</smiles>

Scheme 4

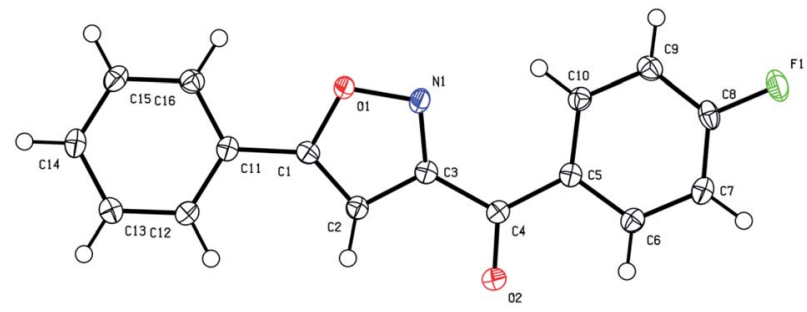

Fig. 1 ORTEP drawing of X-ray structures of (4-fluorophenyl)(5phenylisoxazol-3-yl)methanone (10da, CCDC \#2099762†). The thermal ellipsoids are shown at 50\% probability.

an equimolar mixture of regioisomeric dihydroisoxazoles 12aa and 14aa, formation of which are most likely controlled by electronic and steric factors, respectively (Scheme 5). Reaction of $\mathbf{1 f}$ in the presence of chalcone $\mathbf{1 1 b}$, with enhanced polarization of double bond, resulted in perfect electronicallycontrolled regiochemistry, but was accompanied by oxidative aromatization of the cycloaddition product $\mathbf{1 5 f b}$ (Scheme 5). Finally, reaction of $\mathbf{1 a}$ in the presence of malonic imide $\mathbf{1 6}$ proceeded smoothly, providing bicyclic imide 17a in good yield (Scheme 5).

Since the yields obtained were quite moderate, we decided to investigate the material balance by tracing the byproducts formed in dimerization reactions towards furoxans 8 (Scheme 2). Initially we thought we will be dealing with hydroxamic acids 7, however, benzamides 18 (sometimes in a mixture with benzoic acids 19) were observed and isolated instead. Evidently, under the listed reaction conditions hydroxamic acids 7 undergo facile Lossen rearrangement or competing hydrolysis as shown in Scheme 6. It is believed, that $O$-phosphorylated hydroxamic acid 6 (initially formed after nucleophilic attack on nitrile oxide 2 with ortho-phosphoric acid as shown in Scheme 1 , vide infra) could be re-esterified to afford NO-phosphorylated form 20. The later would undergo Lossen rearrangement into benzoyl isocyanate $\mathbf{2 1}$, which can be hydrolyzed with extrusion of isocyanic acid and formation of benzoic acid 19 (Scheme 6). More commonly, an alternative pathway was operating,

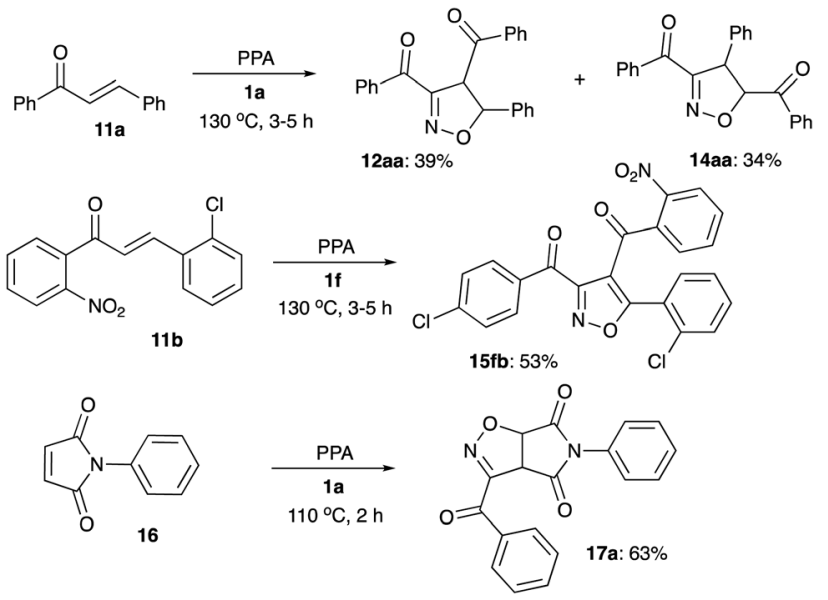

Scheme 5 


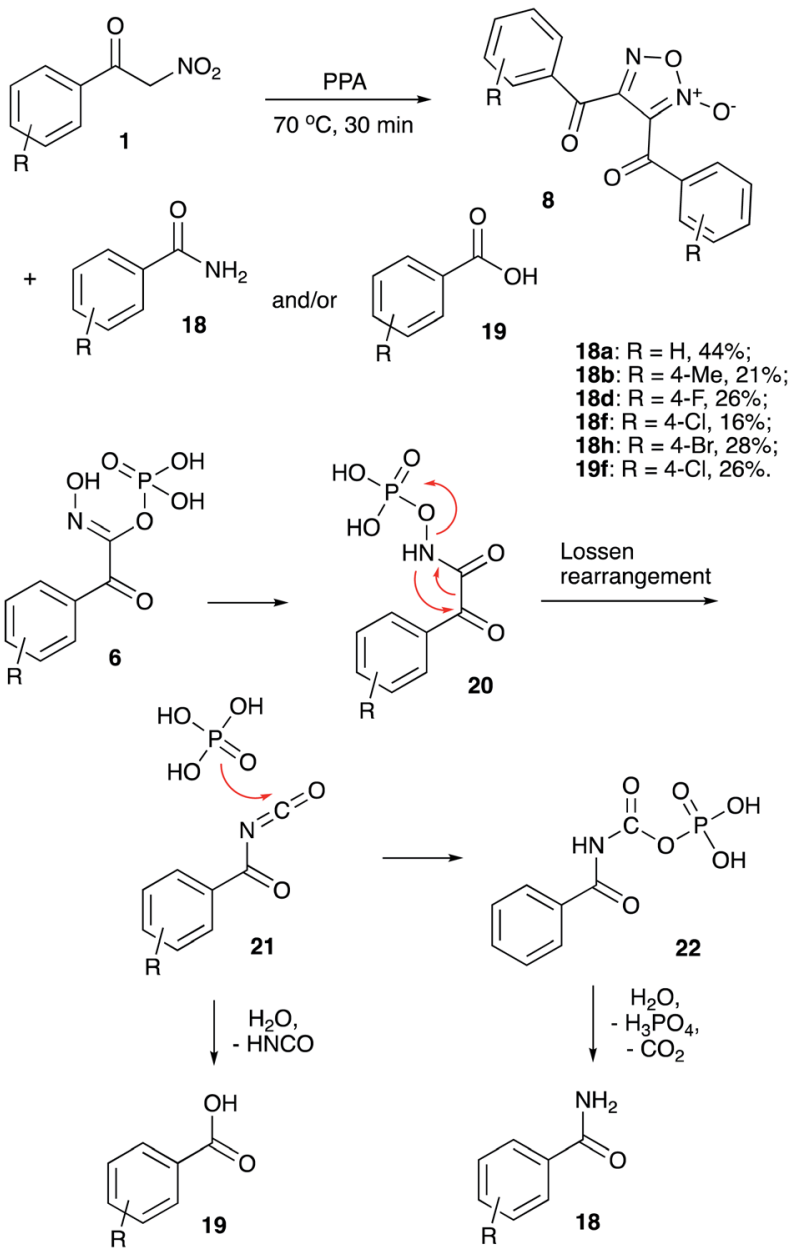

Scheme 6

involving reaction of isocyanate $\mathbf{2 1}$ with phosphoric acid to afford benzoylcarbamic phosphoric anhydride 22, which then underwent hydrolysis to afford benzamide species 18. These compounds were isolated in moderate yield from most of the reaction mixtures along with normal furoxan products $\mathbf{8}$.

\section{Conclusions}

In conclusion, we demonstrated that electrophilic activation of nitroalkanes in the presence of polyphosphoric acid can, at least in some cases, provide nitrile oxide moieties, which likely exists in equilibrium with more ordinary nitronate species. In the presence of PPA, nitrile oxides tend to decompose to hydroxamic acids, but for most stable electron-deficient dipolar species, typical $[3+2]$ cycloaddition reactions can be studied. Nevertheless, formation of hydroxamic acid is a dominant process in most cases, so this reaction cannot be recommended as a preparative method for generation of nitrile oxides.

\section{Experimental}

\section{General}

NMR spectra, ${ }^{1} \mathrm{H}$ and ${ }^{13} \mathrm{C}$ was measured in solutions of $\mathrm{CDCl}_{3}$ or DMSO- $d_{6}$ on Bruker AVANCE-III HD instrument (at 400.40 or
100.61 MHz, respectively). Residual solvent signals were used as internal standards, in DMSO- $d_{6}\left(2.50 \mathrm{ppm}\right.$ for ${ }^{1} \mathrm{H}$, and $40.45 \mathrm{ppm}$ for ${ }^{13} \mathrm{C}$ nuclei) or in $\mathrm{CDCl}_{3}\left(7.26 \mathrm{ppm}\right.$ for ${ }^{1} \mathrm{H}$, and $77.16 \mathrm{ppm}$ for ${ }^{13} \mathrm{C}$ nuclei). HRMS spectra was measured on Bruker maXis impact (electrospray ionization, in MeCN solutions, employing $\mathrm{HCO}_{2} \mathrm{Na}-\mathrm{HCO}_{2} \mathrm{H}$ for calibration). IR spectra was measured on FT-IR spectrometer Shimadzu IRAffinity-1S equipped with an ATR sampling module. Reaction progress, purity of isolated compounds, and $R_{\mathrm{f}}$ values were monitored with TLC on Silufol UV-254 plates. Column chromatography was performed on silica gel (32-63 $\mu \mathrm{m}, 60 \AA$ A pore size). Melting points were measured with Stuart SMP30 apparatus. Polyphosphoric acid samples were prepared by dissolving precisely measured amounts of $\mathrm{P}_{2} \mathrm{O}_{5}$ in $85 \%$ ortho-phosphoric acid. Nitroacetophenones and chalcones were prepared according to the previously published procedures. ${ }^{25-27}$ All other reagents and solvents were purchased from commercial venders and used as received.

1-(4-Ethylphenyl)-2-nitroethan-1-one (1c). 4-Ethylbenzaldehyde ( $1.34 \mathrm{~g}, 10.0 \mathrm{mmol})$, nitromethane $(610 \mathrm{mg}, 10$ $\mathrm{mmol}$ ) and ethanol $(10 \mathrm{~mL})$ were combined in $25 \mathrm{~mL}$ round bottom flask. The reactor was placed into ice bath and aqueous solution of $\mathrm{NaOH}$ ( $425 \mathrm{mg}, 10.5 \mathrm{mmol}$ in $40 \%$ aqueous solution) was added dropwise in such a rate so temperature did not exceed $15{ }^{\circ} \mathrm{C}$. The reaction mixture was stirred for 15 minutes and then quenched with $5 \%$ aqueous solution of acetic acid. The solution was extracted with EtOAc $(5 \times 20 \mathrm{~mL})$ and concentrated in vacuo. Resulting nitroalcohol was employed at the next step without additional purification. Concentrated sulfuric acid $(1.5 \mathrm{~mL})$ was slowly added to an Erlenmeyer flask charged with acetic acid $(14 \mathrm{~mL})$, potassium bichromate $(3.0 \mathrm{~g})$, water $(28 \mathrm{~mL})$, and nitroalcohol cooled in ice bath. The solution was stirred for 24 hours at room temperature, formed precipitate was filtered, washed with cold water, dried, and recrystallized from EtOH. The titled compound was obtained as colorless solid, mp 129.3-131.1 ${ }^{\circ} \mathrm{C}$ (EtOH); yield $1.47 \mathrm{~g}$ $(7.6 \mathrm{mmol}, 76 \%) \cdot R_{\mathrm{f}} 0.29$, EtOAc/hexane $(1: 5, \mathrm{v} / \mathrm{v}) .{ }^{1} \mathrm{H}$ NMR $(400$ MHz, DMSO) $\delta 7.87(\mathrm{~d}, J=8.3 \mathrm{~Hz}, 2 \mathrm{H}), 7.44(\mathrm{~d}, J=8.2 \mathrm{~Hz}, 2 \mathrm{H})$, $6.50(\mathrm{~s}, 2 \mathrm{H}), 2.71(\mathrm{q}, J=7.6 \mathrm{~Hz}, 2 \mathrm{H}), 1.20(\mathrm{t}, J=7.6 \mathrm{~Hz}, 3 \mathrm{H}) .{ }^{13} \mathrm{C}$ NMR (101 MHz, DMSO) $\delta$ 187.9, 151.6, 131.2, 128.7 (2C), 128.5 (2C), 82.8, 28.3, 15.1. IR, $v_{\max } / \mathrm{cm}^{-1}: 3027,2974,2935,1961$, $1933,1694,1607,1549,1328,1229,1185$. HRMS (ES TOF) calcd for $\mathrm{C}_{10} \mathrm{H}_{11} \mathrm{NNaO}_{3}\left([\mathrm{M}+\mathrm{Na}]^{+}\right)$: 216.0631, found $216.0633(-0.7$ ppm).

3,4-Dibenzoyl-1,2,5-oxadiazole 2-oxide (8a). General method for synthesis of furoxans. 2-Nitroacetophenone $1 \mathrm{a}^{27}(165 \mathrm{mg}$, $1.00 \mathrm{mmol}$ ), was combined with $2.0 \mathrm{~g}$ of polyphosphoric acid ( $87 \mathrm{wt} \%$ of $\mathrm{P}_{2} \mathrm{O}_{5}$ ). The reaction mixture was stirred at $70{ }^{\circ} \mathrm{C}$ for 30 minutes. Then the mixture was diluted with water $(40 \mathrm{~mL})$ and quenched with aqueous ammonia $\left(20 \%\right.$ solution in $\mathrm{H}_{2} \mathrm{O}, 15$ $\mathrm{mL})$. The material was extracted with ethyl acetate $(4 \times 15 \mathrm{~mL})$ and combined organic phases were washed with brine. The solution was concentrated in vacuo, and the residue was purified by column chromatography on silica gel eluting EtOAc/PE, $(1: 4 \mathrm{v} / \mathrm{v})$ to give pure furoxan. Colorless oil, $R_{\mathrm{f}} 0.5$, EtOAc/ hexane $(1: 4, \mathrm{v} / \mathrm{v})$. Yield $72 \mathrm{mg}(0.24 \mathrm{mmol}, 49 \%) .{ }^{1} \mathrm{H}$ NMR $\left(400 \mathrm{MHz}, \mathrm{CDCl}_{3}\right) \delta 8.25-8.18(\mathrm{~m}, 2 \mathrm{H}), 7.93-7.80(\mathrm{~m}, 2 \mathrm{H}), 7.76-$ 
$7.65(\mathrm{~m}, 2 \mathrm{H}), 7.60-7.49(\mathrm{~m}, 4 \mathrm{H}) .{ }^{13} \mathrm{C} \mathrm{NMR}\left(101 \mathrm{MHz}, \mathrm{CDCl}_{3}\right.$ ) $\delta 182.2,180.9,154.8,135.9,135.8,134.3,134.3,131.0$ (2C), 130.1 (2C), 129.7 (2C), 129.5 (2C), 112.1. IR, $v_{\max } / \mathrm{cm}^{-1}: 1735,1684$, 1602, 1559, 1455, 1419, 1325, 1246, 1178, 1101. HRMS (ES TOF) calcd for $\mathrm{C}_{16} \mathrm{H}_{10} \mathrm{~N}_{2} \mathrm{NaO}_{4}\left([\mathrm{M}+\mathrm{Na}]^{+}\right): 317.0533$, found 317.0532 (0.4 ppm).

Benzamide (18a) ${ }^{28}$ was also isolated from the same reaction mixture as colorless solid. Yield $53 \mathrm{mg}$ (0.44 mmol, 44\%). ${ }^{1} \mathrm{H}$ NMR (400 MHz, $\mathrm{CDCl}_{3}$ ) $\delta 7.84(\mathrm{dt}, J=7.1,1.4 \mathrm{~Hz}, 2 \mathrm{H}), 7.59-7.50$ $(\mathrm{m}, 1 \mathrm{H}), 7.46(\mathrm{t}, J=7.5 \mathrm{~Hz}, 2 \mathrm{H}), 6.35(\mathrm{~s}, 2 \mathrm{H}) .{ }^{13} \mathrm{C} \mathrm{NMR}(101 \mathrm{MHz}$, $\left.\mathrm{CDCl}_{3}\right) \delta 170.2,133.8,132.4,129.1$ (2C), 127.8 (2C).

3,4-Bis(4-methylbenzoyl)-1,2,5-oxadiazole 2-oxide (8b). Product $\mathbf{8 b}$ was obtained via the method described for compound 8a, employing 2-nitro-4'-methylacetophenone $\mathbf{1 b}^{\mathbf{2 7}}$ (179 $\mathrm{mg}, 1.00 \mathrm{mmol}$ ), and purified by column chromatography (eluent EtOAc/PE 1 : 5). Yellow solid, mp $118-119.5{ }^{\circ} \mathrm{C}(\mathrm{EtOH})$, $\mathrm{lit}^{29} \mathrm{mp} 124{ }^{\circ} \mathrm{C}(\mathrm{EtOH})$; yield $83 \mathrm{mg}(0.26 \mathrm{mmol}, 52 \%) . R_{\mathrm{f}} 0.46$, EtOAc/hexane $(1: 5, \mathrm{v} / \mathrm{v}) .{ }^{1} \mathrm{H}$ NMR $\left(400 \mathrm{MHz} \mathrm{CDCl}_{3}\right) \delta 8.11(\mathrm{~d}, J$ $=8.3 \mathrm{~Hz}, 2 \mathrm{H}), 7.76(\mathrm{~d}, J=8.3 \mathrm{~Hz}, 2 \mathrm{H}), 7.33(\mathrm{dd}, J=13.4,8.3 \mathrm{~Hz}$, $4 \mathrm{H}), 2.45$ (d, $J=10.1 \mathrm{~Hz}, 6 \mathrm{H}) .{ }^{13} \mathrm{C} \mathrm{NMR} \mathrm{(101} \mathrm{MHz,} \mathrm{CDCl}_{3}$ ) $\delta 181.3,180.0,154.6,147.0,146.8,131.5,131.4,130.7$ (2C), 130.0 (2C), 129.9 (2C), 129.8 (2C), 111.9, 22.0 (2C). IR, $v_{\max } / \mathrm{cm}^{-1}: 1675$, $1646,1600,1470,1328,1251,1181,1123,1104$. HRMS (ES TOF) calcd for $\mathrm{C}_{18} \mathrm{H}_{14} \mathrm{~N}_{2} \mathrm{NaO}_{4}\left([\mathrm{M}+\mathrm{Na}]^{+}\right): 345.0846$, found 345.0840 (1.7 ppm).

4-Methylbenzamide $(\mathbf{1 8 b})^{28}$ was also isolated from the same reaction mixture as colorless solid. Yield $28 \mathrm{mg}(0.21 \mathrm{mmol}$, 21\%). ${ }^{1} \mathrm{H}$ NMR (400 MHz, DMSO- $\left.d_{6}\right) \delta 7.90(\mathrm{~s}, 1 \mathrm{H}), 7.77(\mathrm{~d}, J=$ $8.2 \mathrm{~Hz}, 2 \mathrm{H}), 7.29(\mathrm{~s}, 1 \mathrm{H}), 7.24(\mathrm{~d}, J=8.0 \mathrm{~Hz}, 2 \mathrm{H}), 2.34(\mathrm{~s}, 3 \mathrm{H}) .{ }^{13} \mathrm{C}$ NMR (101 MHz, DMSO- $\left.d_{6}\right) \delta 167.8,141.1,131.5,128.8$ (2C), 127.6 (2C), 21.0.

3,4-Bis(4-ethylbenzoyl)-1,2,5-oxadiazole 2-oxide (8c). Product 8c was obtained via the method described for compound 8a, employing 2-nitro-4'-ethylacetophenone 1c (193 mg, 1.00 $\mathrm{mmol}$ ), and purified by column chromatography (eluent EtOAc/ PE, $1: 4)$. Colorless oil, $R_{\mathrm{f}} 0.60$, EtOAc/hexane $(1: 5, \mathrm{v} / \mathrm{v})$. Yield $86 \mathrm{mg}$ (0.25 mmol, 49\%). ${ }^{1} \mathrm{H}$ NMR (400 MHz, $\left.\mathrm{CDCl}_{3}\right) \delta 8.18-8.07$ (m, 2H), 7.83-7.70 (m, 2H), 7.36 (dd, $J=13.6,8.1 \mathrm{~Hz}, 4 \mathrm{H}), 2.75$ $(\mathrm{dq}, J=9.8,7.6 \mathrm{~Hz}, 4 \mathrm{H}), 1.28$ (q, $J=7.8 \mathrm{~Hz}, 6 \mathrm{H}) .{ }^{13} \mathrm{C} \mathrm{NMR}(101$ $\left.\mathrm{MHz}, \mathrm{CDCl}_{3}\right) \delta 181.5,180.1,154.7,153.2,152.9,131.8,131.7$, 131.0 (2C), 130.1 (2C), 128.9 (2C), 128.7 (2C), 112.1, 29.4, 29.3, 15.1, 15.1. IR, $v_{\max } / \mathrm{cm}^{-1}: 2964,2877,1733,1595,1460,1427$, 1383, 1337, 1272, 1147, 1096. HRMS (ES TOF) calcd for $\mathrm{C}_{20} \mathrm{H}_{18} \mathrm{~N}_{2} \mathrm{NaO}_{4}\left([\mathrm{M}+\mathrm{Na}]^{+}\right): 373.1159$, found 373.1151 (2.0 ppm).

3,4-Bis(4-fluorobenzoyl)-1,2,5-oxadiazole 2-oxide (8d). Product 8d was obtained via the method described for compound 8a, employing 2-nitro-4'-fluoroacetophenone $\mathbf{1 d}^{\mathbf{2 7}}$ (183 $\mathrm{mg}, 1.00 \mathrm{mmol}$ ), and purified by column chromatography (eluent EtOAc/PE, $1: 4$ ). Yellow oil, $R_{\mathrm{f}} 0.53$, EtOAc/hexane $(1: 5$, $\mathrm{v} / \mathrm{v})$. Yield $58 \mathrm{mg}(0.18 \mathrm{mmol}, 35 \%) .{ }^{1} \mathrm{H} \mathrm{NMR}\left(400 \mathrm{MHz}, \mathrm{CDCl}_{3}\right.$ ) $\delta$ 8.40-8.34 (m, 2H), 8.03-7.97 (m, 2H), 7.32 (m, $J=11.1,8.4 \mathrm{~Hz}$, 4H). ${ }^{13} \mathrm{C} \mathrm{NMR}\left(101 \mathrm{MHz}, \mathrm{CDCl}_{3}\right) \delta 180.2,178.9,167.30(\mathrm{~d}, J=$ $259.7 \mathrm{~Hz}$ ), 167.28 (d, $J=259.3 \mathrm{~Hz}), 154.3,133.7$ (d, $J=9.9 \mathrm{~Hz}$, 2C), 132.8 (d, $J=10.3 \mathrm{~Hz}, 2 \mathrm{C}), 130.3(\mathrm{~d}, J=2.9 \mathrm{~Hz}), 130.2(\mathrm{~d}, J=$ $2.9 \mathrm{~Hz}), 116.9$ (d, $J=22.4 \mathrm{~Hz}, 2 \mathrm{C}), 116.6$ (d, $J=22.0 \mathrm{~Hz}, 2 \mathrm{C})$, 111.7. IR, $v_{\text {max }} / \mathrm{cm}^{-1}: 1769,1684,1663,1593,1508,1472,1414$,
1325, 1236, 1157, 1101. HRMS (ES TOF) calcd for $\mathrm{C}_{16} \mathrm{H}_{8} \mathrm{~F}_{2} \mathrm{~N}_{2}$ $\mathrm{NaO}_{4}\left([\mathrm{M}+\mathrm{Na}]^{+}\right): 353.0344$, found 353.0338 (1.7 ppm).

4-Fluorobenzamide $(\mathbf{1 8 b})^{\mathbf{2 8}}$ was also isolated from the same reaction mixture as colorless solid. Yield $36 \mathrm{mg}(0.26 \mathrm{mmol}$, $26 \%$ ). ${ }^{1} \mathrm{H}$ NMR (400 MHz, DMSO- $\left.d_{6}\right) \delta 8.01(\mathrm{~s}, 1 \mathrm{H}), 7.97-7.87(\mathrm{~m}$, $2 \mathrm{H}), 7.41$ (s, 1H), 7.32-7.19 (m, 2H). ${ }^{13} \mathrm{C}$ NMR (101 MHz, DMSO) $\delta 166.0(\mathrm{~d}, J=164.9 \mathrm{~Hz}), 162.7,130.8(\mathrm{~d}, J=3.0 \mathrm{~Hz}) 130.2(\mathrm{~d}, J=$ $9.0 \mathrm{~Hz}, 2 \mathrm{C}), 115.2$ (d, $J=21.6 \mathrm{~Hz}, 2 \mathrm{C})$.

3,4-Bis(2-fluorobenzoyl)-1,2,5-oxadiazole 2-oxide (8e). Product 8e was obtained via the method described for compound 8a, employing 2-nitro-2'-fluoroacetophenone $\mathbf{1 e}^{\mathbf{2 6}}$ (183 $\mathrm{mg}, 1.00 \mathrm{mmol}$ ), and purified by column chromatography (eluent EtOAc/PE $1: 4$ ). Yellow oil, $R_{\mathrm{f}} 0.32$, EtOAc/hexane $(1: 4$, $\mathrm{v} / \mathrm{v})$. Yield $81 \mathrm{mg}(0.25 \mathrm{mmol}, 49 \%) .{ }^{1} \mathrm{H}$ NMR (400 MHz, $\mathrm{CDCl}_{3}$ ) $\delta 7.92(\mathrm{td}, J=7.8,1.8 \mathrm{~Hz}, 1 \mathrm{H}), 7.82(\mathrm{td}, J=7.4,1.8 \mathrm{~Hz}, 1 \mathrm{H}), 7.70-$ $7.61(\mathrm{~m}, 2 \mathrm{H}), 7.36-7.29(\mathrm{~m}, 2 \mathrm{H}), 7.17$ (m, $J=25.1,11.0,8.4$, $1.2 \mathrm{~Hz}, 2 \mathrm{H}) .{ }^{13} \mathrm{C}$ NMR (101 MHz, $\left.\mathrm{CDCl}_{3}\right) \delta 180.1,177.1,162.0(\mathrm{~d}$, $J=259.0 \mathrm{~Hz}), 161.6(\mathrm{~d}, J=256.4 \mathrm{~Hz}), 154.4,136.8(\mathrm{~d}, J=7.6 \mathrm{~Hz})$, $136.7(\mathrm{~d}, J=7.4 \mathrm{~Hz}), 131.5,131.1,125.2(\mathrm{~d}, J=3.3 \mathrm{~Hz}), 124.8(\mathrm{~d}, J$ $=3.7 \mathrm{~Hz}), 123.4(\mathrm{~d}, J=10.3 \mathrm{~Hz}), 123.2(\mathrm{~d}, J=11.0 \mathrm{~Hz}), 117.0(\mathrm{~d}, J$ $=21.6 \mathrm{~Hz}), 116.6(\mathrm{~d}, J=22.0 \mathrm{~Hz}), 112.7 . \mathrm{IR}, v_{\max } / \mathrm{cm}^{-1}: 1655$, 1602, 1458, 1439, 1330, 1238, 1181, 1159, 1099, 1041. HRMS (ES TOF) calcd for $\mathrm{C}_{16} \mathrm{H}_{8} \mathrm{~F}_{2} \mathrm{~N}_{2} \mathrm{NaO}_{4}\left([\mathrm{M}+\mathrm{Na}]^{+}\right)$: 353.0344, found 353.0334 (3.0 ppm).

3,4-Bis(4-chlorobenzoyl)-1,2,5-oxadiazole 2-oxide (8f). Product 8f was obtained via the method described for compound 8a, employing 2-nitro-4'-chloroacetophenone $\mathbf{1 f}^{\mathbf{2 7}}$ (199 $\mathrm{mg}, 1.00 \mathrm{mmol}$ ), and purified by column chromatography (eluent EtOAc/PE $1: 4)$. Colorless solid, mp 120-121 ${ }^{\circ} \mathrm{C}(\mathrm{EtOH})$, $\mathrm{lit}^{30} \mathrm{mp} 124{ }^{\circ} \mathrm{C} ; R_{\mathrm{f}} 0.57$, EtOAc/hexane $(1: 6, \mathrm{v} / \mathrm{v})$. Yield $92 \mathrm{mg}$ (0.25 mmol, 51\%). ${ }^{1} \mathrm{H}$ NMR (400 $\left.\mathrm{MHz}, \mathrm{CDCl}_{3}\right) \delta 8.18(\mathrm{~d}, J=$ $8.7 \mathrm{~Hz}, 2 \mathrm{H}), 7.80(\mathrm{~d}, J=8.6 \mathrm{~Hz}, 2 \mathrm{H}), 7.53(\mathrm{~m}, J=12.0,8.7 \mathrm{~Hz}$, $4 \mathrm{H}) .{ }^{13} \mathrm{C} \mathrm{NMR}\left(101 \mathrm{MHz}, \mathrm{CDCl}_{3}\right) \delta 180.5,179.2,154.0,142.41$, 142.39, 132.01, 132.00, 131.9 (2C), 131.0 (2C), 129.8 (2C), 129.5 (2C), 111.4. IR, $v_{\max } / \mathrm{cm}^{-1}$ : 1773, 1682, 1610, 1586, 1472, 1405, 1323, 1243, 1176, 1089. HRMS (ES TOF) calcd for $\mathrm{C}_{16} \mathrm{H}_{8} \mathrm{Cl}_{2} \mathrm{~N}_{2}$ $\mathrm{NaO}_{4}\left([\mathrm{M}+\mathrm{Na}]^{+}\right): 384.9753$, found $384.9760(-1.8 \mathrm{ppm})$.

4-Chlorobenzamide $(\mathbf{1 8 f})^{\mathbf{2 8}}$ and 4-chlorobenzoic acid (19f $)^{\mathbf{3 1}}$ were also isolated from the same reaction mixture as colorless solid. 18f: yield $25 \mathrm{mg}$ (0.16 mmol, 16\%). ${ }^{1} \mathrm{H}$ NMR (400 MHz, DMSO- $\left.d_{6}\right) \delta 8.06$ (br. s, 1H), 7.93-7.84 (m, 2H), 7.55-7.50 (m, 2H), 7.49 (br. s, $1 \mathrm{H}) .{ }^{13} \mathrm{C}$ NMR (101 MHz, DMSO- $\left.d_{6}\right) \delta 166.9$, 136.1, 133.1, 129.5 (2C), 128.4 (2C). 19f: yield $41 \mathrm{mg}$ (0.26 mmol, 26\%). ${ }^{1} \mathrm{H}$ NMR (400 MHz, DMSO- $d_{6}$ ) $\delta 13.19$ (br. s, $\left.1 \mathrm{H}\right), 7.93$ (d, $J$ $=8.6 \mathrm{~Hz}, 2 \mathrm{H}), 7.55(\mathrm{~d}, J=8.5 \mathrm{~Hz}, 2 \mathrm{H}) .{ }^{13} \mathrm{C} \mathrm{NMR}(101 \mathrm{MHz}$, DMSO- $\left.d_{6}\right) \delta 166.5,137.8,131.2$ (2C), 129.7, 128.8 (2C).

3,4-Bis(3-chlorobenzoyl)-1,2,5-oxadiazole 2-oxide (8g). Product $\mathbf{8 g}$ was obtained via the method described for compound 8a, employing 2-nitro-3'-chloroacetophenone $\mathbf{1 g}^{\mathbf{2 7}}$ (199 $\mathrm{mg}, 1.00 \mathrm{mmol}$ ), and purified by column chromatography (eluent EtOAc/PE, $1: 4)$. Yellow oil; $R_{\mathrm{f}} 0.49$, EtOAc/hexane $(1: 4$, $\mathrm{v} / \mathrm{v})$. Yield $102 \mathrm{mg}(0.28 \mathrm{mmol}, 56 \%) .{ }^{1} \mathrm{H} \mathrm{NMR}\left(400 \mathrm{MHz}, \mathrm{CDCl}_{3}\right)$ $\delta 8.17(\mathrm{~m}, J=1.9 \mathrm{~Hz}, 1 \mathrm{H}), 8.12(\mathrm{~m}, J=7.8,1.4 \mathrm{~Hz}, 1 \mathrm{H}), 7.84(\mathrm{~m}, J$ $=1.9 \mathrm{~Hz}, 1 \mathrm{H}), 7.73-7.64(\mathrm{~m}, 3 \mathrm{H}), 7.50(\mathrm{~m}, J=15.8,7.9 \mathrm{~Hz}, 2 \mathrm{H})$. ${ }^{13} \mathrm{C}$ NMR $\left(101 \mathrm{MHz}, \mathrm{CDCl}_{3}\right) \delta 180.5,179.2,153.8,135.8,135.50$, 135.46, 135.3, 135.2, 135.11, 130.6, 130.4, 130.3, 129.4, 128.7, 127.8, 111.1. IR, $v_{\max } / \mathrm{cm}^{-1}: 1754,1687,1614,1571,1467,1419$, 
1323, 1236, 1079. HRMS (ES TOF) calcd for $\mathrm{C}_{16} \mathrm{H}_{8} \mathrm{Cl}_{2} \mathrm{~N}_{2} \mathrm{NaO}_{4}([\mathrm{M}$ $\left.+\mathrm{Na}]^{+}\right): 384.9753$, found $384.9762(-2.2 \mathrm{ppm})$.

3,4-Bis(4-bromobenzoyl)-1,2,5-oxadiazole 2-oxide (8h). Product $\mathbf{8 h}$ was obtained via the method described for compound 8a, employing 2-nitro-4'-bromoacetophenone $\mathbf{1 h}^{\mathbf{2 7}}$ (244 $\mathrm{mg}, 1.00 \mathrm{mmol}$ ), and purified by column chromatography (eluent EtOAc/PE, $1: 4$ ). Yellowish solid, mp 135.9-136.7 ${ }^{\circ} \mathrm{C}$ (EtOAc), $R_{\mathrm{f}}$ 0.57, EtOAc/hexane $(1: 4, \mathrm{v} / \mathrm{v})$. Yield $90 \mathrm{mg}$ (0.20 mmol, 40\%). ${ }^{1} \mathrm{H}$ NMR (400 MHz, $\mathrm{CDCl}_{3}$ ) $\delta 8.12-8.05(\mathrm{~m}$, $2 \mathrm{H}), 7.76-7.64(\mathrm{~m}, 6 \mathrm{H}) .{ }^{13} \mathrm{C}$ NMR $\left(101 \mathrm{MHz}, \mathrm{CDCl}_{3}\right) \delta$ 180.7, 179.4, 154.0, 132.7 (2C), 132.5 (2C), 132.4 (2C), 131.9 (2C), 131.3 (2C), 131.0 (2C), 111.3. IR, $v_{\max } / \mathrm{cm}^{-1}: 928,1012,1067,1173$, 1212, 1251, 1325, 1395, 1467, 1581, 1612, 1655, 1689, 3089. HRMS (ES TOF) calcd for $\mathrm{C}_{16} \mathrm{H}_{8} \mathrm{Br}_{2} \mathrm{~N}_{2} \mathrm{NaO}_{4}\left([\mathrm{M}+\mathrm{Na}]^{+}\right)$: 472.8743, found 472.8741 (0.4 ppm).

4-Bromobenzamide $(\mathbf{1 8 h})^{28}$ was also isolated from the same reaction mixture as colorless solid. Yield $56 \mathrm{mg}(0.28 \mathrm{mmol}$, $28 \%) .{ }^{1} \mathrm{H}$ NMR (400 MHz, DMSO- $\left.d_{6}\right) \delta 8.06(\mathrm{~s}, 1 \mathrm{H}), 7.84-7.78(\mathrm{~m}$, 2H), 7.70-7.63 (m, 2H), 7.48 (s, 1H). ${ }^{13} \mathrm{C}$ NMR (101 MHz, DMSO) $\delta 167.4,133.8,131.7$ (2C), 130.1 (2C), 125.5 .

3,4-Bis(ethoxycarbonyl)-1,2,5-oxadiazole 2-oxide (8i). Product 8i was obtained via the method described for compound 8a, employing ethyl nitroacetate (133 mg, 1.00 $\mathrm{mmol}$ ), and purified by column chromatography (eluent EtOAc/ PE, $1: 4)$. Colorless oil, $R_{\mathrm{f}} 0.46$, EtOAc/hexane $(1: 4, \mathrm{v} / \mathrm{v})$. Yield $49 \mathrm{mg}$ (0.21 mmol, 43\%). ${ }^{1} \mathrm{H}$ NMR (400 MHz, DMSO- $\left.d_{6}\right) \delta 4.45$ $(\mathrm{qd}, J=7.1,1.0 \mathrm{~Hz}, 2 \mathrm{H}), 4.36(\mathrm{qd}, J=7.1,1.1 \mathrm{~Hz}, 2 \mathrm{H}), 1.33(\mathrm{td}, J$ $=7.1,1.0 \mathrm{~Hz}, 3 \mathrm{H}), 1.27(\mathrm{td}, J=7.1,1.1 \mathrm{~Hz}, 3 \mathrm{H}) .{ }^{13} \mathrm{C} \mathrm{NMR}(101$ MHz, DMSO) $\delta 156.5,154.8,148.8,107.4,63.5,63.1,13.72,13.7$. IR, $v_{\text {max }} / \mathrm{cm}^{-1}:$ 2988, 1742, 1619, 1472, 1373, 1335, 1246, 1195, 1063, 1022. HRMS (ES TOF) calcd for $\mathrm{C}_{8} \mathrm{H}_{10} \mathrm{~N}_{2} \mathrm{NaO}_{6}\left([\mathrm{M}+\mathrm{Na}]^{+}\right)$: 253.0431, found 253.0426 (2.1 ppm).

Procedure for preparation of cyclization products of hydrazine hydrate with 2-nitroethan-1-one, 4,7-diphenyl-[1,2,5]oxadiazolo[3,4- $d]$ pyridazine-1-oxide (13a). 2-Nitroacetophenone 1a (165 mg, $1.00 \mathrm{mmol}$ ), hydrazine monohydrate (65\% solution in water, $154 \mathrm{mg}, 150 \mu \mathrm{L}, 2.00 \mathrm{mmol}$ ) were added to $2 \mathrm{~g}$ of $87 \%$ polyphosphoric acid. The mixture was stirred at $110^{\circ} \mathrm{C}$ for $2 \mathrm{~h}$ and then diluted with water $(40 \mathrm{~mL})$ and quenched with aqueous ammonia (20\% solution in $\mathrm{H}_{2} \mathrm{O}, 15 \mathrm{~mL}$ ). Reaction mixture was extracted with ethyl acetate $(4 \times 15 \mathrm{~mL})$. Combined organic layers were washed with brine and concentrated in vacuo. The residue was purified by column chromatography on silica gel (eluent: EtOAc: PE, 1:4 v/v) affording the titled product as orange solid, mp $199-201{ }^{\circ} \mathrm{C}$ (EtOAc), lit $^{30} \mathrm{mp} 210{ }^{\circ} \mathrm{C}$; $R_{\mathrm{f}} 0.44$, EtOAc/hexane (1: 4, v/v). Yield $31 \mathrm{mg}(0.11 \mathrm{mmol}, 21 \%)$. ${ }^{1} \mathrm{H}$ NMR (400 MHz, $\mathrm{CDCl}_{3}$ ) $\delta 8.68-8.60(\mathrm{~m}, 2 \mathrm{H}), 8.00-7.90(\mathrm{~m}$, 2H), 7.68-7.56 (m, 6H). ${ }^{13} \mathrm{C}$ NMR (101 MHz, $\left.\mathrm{CDCl}_{3}\right) \delta$ 150.4, 148.3, 145.1, 132.6, 131.9, 131.8, 130.3, 129.6 (2C), 129.3 (2C), 129.2 (2C), 128.7 (2C), 106.9. IR, $v_{\max } / \mathrm{cm}^{-1}: 2974,2882,1600$, 1508, 1460, 1429, 1388, 1263, 1147, 1089, 1048. HRMS (ES TOF) calcd for $\mathrm{C}_{16} \mathrm{H}_{10} \mathrm{~N}_{4} \mathrm{NaO}_{2}\left([\mathrm{M}+\mathrm{Na}]^{+}\right)$: 313.0696, found 313.0691 (1.7 ppm).

5-Phenyl-3-benzoylisoxazole. General method for synthesis of isoxazoles (10aa). 2-Nitroacetophenone $1^{27}$ (165 mg, 1.00 $\mathrm{mmol}$ ) and phenylacetylene $9 \mathrm{a}(102 \mathrm{mg}, 1.00 \mathrm{mmol})$ were added to $2.0 \mathrm{~g}$ of $87 \%$ polyphosphoric acid. The mixture was stirred at
$110{ }^{\circ} \mathrm{C}$ for $2 \mathrm{~h}$ and then diluted with water $(40 \mathrm{~mL})$ and quenched with aqueous ammonia (20\% solution in $\mathrm{H}_{2} \mathrm{O}, 15$ $\mathrm{mL})$. Reaction mixture was extracted with ethyl acetate $(4 \times 15$ $\mathrm{mL}$ ). Combined organic layers were washed with brine and concentrated in vacuo. The residue was purified by column chromatography on silica gel (eluent: benzene/hexane, $1: 2 \mathrm{v} / \mathrm{v}$ ) to afford the titled compound as yellowish solid, mp $85.4-86{ }^{\circ} \mathrm{C}$ (EtOH), lit $^{32} \mathrm{mp} 88-90{ }^{\circ} \mathrm{C}, R_{\mathrm{f}} 0.46$, benzene/hexane $(1: 2, \mathrm{v} / \mathrm{v})$. Yield $65 \mathrm{mg}$ (0.26 mmol, 26\%). ${ }^{1} \mathrm{H}$ NMR (400 $\mathrm{MHz}, \mathrm{CDCl}_{3}$ ) $\delta 8.38-8.32(\mathrm{~m}, 2 \mathrm{H}), 7.86(\mathrm{~m}, J=7.8,1.9 \mathrm{~Hz}, 2 \mathrm{H}), 7.67(\mathrm{~m}, J=$ $7.4 \mathrm{~Hz}, 1 \mathrm{H}), 7.53(\mathrm{~m}, J=17.5,7.9 \mathrm{~Hz}, 5 \mathrm{H}), 7.06(\mathrm{~s}, 1 \mathrm{H}) .{ }^{13} \mathrm{C} \mathrm{NMR}$ $\left(101 \mathrm{MHz}, \mathrm{CDCl}_{3}\right) \delta 185.9,170.8,162.4,135.8,134.1,130.8$, 130.7 (2C), 129.2 (2C), 128.6 (2C), 126.7, 126.0 (2C), 100.3. IR, $v_{\max } / \mathrm{cm}^{-1}: 1655,1598,1573,1451,1426,1236,1181,1147,1101$, 1075. HRMS (ES TOF) calcd for $\mathrm{C}_{16} \mathrm{H}_{11} \mathrm{NNaO}_{2}\left([\mathrm{M}+\mathrm{Na}]^{+}\right)$: 272.0682, found 272.0678 (1.3 ppm).

5-Pentyl-3-benzoylisoxazole (10ab). Product 10ab was obtained via the method described for compound 10aa, employing 2-nitroacetophenone $1 \mathrm{a}^{27}$ (165 $\mathrm{mg}, 1.00 \mathrm{mmol}$ ) and hept-1-yne 9b (96 mg, $1.00 \mathrm{mmol}$ ) and purified by column chromatography (eluent EtOAc/PE $1: 4$ ). Colorless oil; $R_{\mathrm{f}}$ 0.72, EtOAc/ hexane $(1: 4, \mathrm{v} / \mathrm{v})$. Yield $68 \mathrm{mg}(0.28 \mathrm{mmol}, 28 \%) .{ }^{1} \mathrm{H}$ NMR $\left(400 \mathrm{MHz}, \mathrm{CDCl}_{3}\right) \delta 8.33-8.26(\mathrm{~m}, 2 \mathrm{H}), 7.67-7.61(\mathrm{~m}, 1 \mathrm{H}), 7.54-$ $7.48(\mathrm{~m}, 2 \mathrm{H}), 6.52(\mathrm{~s}, 1 \mathrm{H}), 2.86-2.80(\mathrm{~m}, 2 \mathrm{H}), 1.80-1.71(\mathrm{~m}, 2 \mathrm{H})$, 1.42-1.34 (m, 4H), 0.95-0.89 (m, 3H). ${ }^{13} \mathrm{C}$ NMR (101 MHz, $\left.\mathrm{CDCl}_{3}\right) \delta 185.2,173.8,160.8,134.9,132.9,129.6$ (2C), $127.5(2 \mathrm{C})$, 100.6, 30.1, 26.1, 25.6, 21.3, 12.9. IR, $v_{\max } / \mathrm{cm}^{-1}: 2930,1774$, 1660, 1602, 1455, 1434, 1258, 1239, 1181, 1104. HRMS (ES TOF) calcd for $\mathrm{C}_{15} \mathrm{H}_{17} \mathrm{NNaO}_{2}\left([\mathrm{M}+\mathrm{Na}]^{+}\right)$: 266.1151, found 266.1145 (2.6 ppm).

5-(Bromomethyl)-3-benzoylisoxazole (10ac). Product 10ac was obtained via the method described for compound 10aa, employing 2-nitroacetophenone $\mathbf{1 a}^{27}(165 \mathrm{mg}, 1.00 \mathrm{mmol})$ and propargyl bromide 9c (119 $\mathrm{mg}, 1.00 \mathrm{mmol})$ and purified by column chromatography (eluent EtOAc/PE $1: 4$ ). Yellow solid, $\mathrm{mp} 62-65{ }^{\circ} \mathrm{C} ; R_{\mathrm{f}} 0.73$, EtOAc/hexane $(1: 4, \mathrm{v} / \mathrm{v})$. Yield $82 \mathrm{mg}$ (0.31 mmol, 31\%). ${ }^{1} \mathrm{H}$ NMR (400 MHz, $\left.\mathrm{CDCl}_{3}\right) \delta 8.31-8.28(\mathrm{~m}$, $2 \mathrm{H}), 7.68-7.63(\mathrm{~m}, 1 \mathrm{H}), 7.55-7.50(\mathrm{~m}, 2 \mathrm{H}), 6.85(\mathrm{~d}, J=0.6 \mathrm{~Hz}$, $1 \mathrm{H}), 4.54(\mathrm{~d}, J=0.6 \mathrm{~Hz}, 2 \mathrm{H}) .{ }^{13} \mathrm{C} \mathrm{NMR}\left(101 \mathrm{MHz}, \mathrm{CDCl}_{3}\right) \delta 185.3$, 168.5, 162.3, 135.6, 134.4, 130.8 (2C), 128.8 (2C), 104.9, 18.1. IR, $v_{\max } / \mathrm{cm}^{-1}: 3152,2959,1766,1645,1599,1578,1452,1291,1224$, 1187, 999. HRMS (ES TOF) calcd for $\mathrm{C}_{11} \mathrm{H}_{8} \mathrm{BrNaNO}_{2}\left([\mathrm{M}+\mathrm{Na}]^{+}\right)$: 287.9631 , found 287.9624 (2.2 ppm).

3-(4-Ethylbenzoyl)-5-phenylisoxazole (10ca). Product 10ca was obtained via the method described for compound 10aa, employing 2-nitro-4'-ethylacetophenone $1 \mathrm{c}$ (193 mg, $1.00 \mathrm{mmol})$ and phenylacetylene 9a (102 $\mathrm{mg}, 1.00 \mathrm{mmol})$ and purified by column chromatography (eluent EtOAc : PE $1: 4)$. White solid, mp 84-85 ${ }^{\circ} \mathrm{C}($ EtOH $), R_{\mathrm{f}} 0.49$, benzene/hexane $(1: 2, \mathrm{v} / \mathrm{v})$. Yield $105 \mathrm{mg}$ (0.38 mmol, 38\%). ${ }^{1} \mathrm{H}$ NMR $\left(400 \mathrm{MHz}, \mathrm{CDCl}_{3}\right) \delta 8.28(\mathrm{~d}, J$ $=8.3 \mathrm{~Hz}, 2 \mathrm{H}), 7.85(\mathrm{dd}, J=7.8,1.8 \mathrm{~Hz}, 2 \mathrm{H}), 7.55-7.47(\mathrm{~m}, 3 \mathrm{H})$, $7.36(\mathrm{~d}, J=8.3 \mathrm{~Hz}, 2 \mathrm{H}), 7.04(\mathrm{~s}, 1 \mathrm{H}), 2.75$ (q, $J=7.6 \mathrm{~Hz}, 2 \mathrm{H}), 1.29$ $(\mathrm{t}, J=7.6 \mathrm{~Hz}, 3 \mathrm{H}) .{ }^{13} \mathrm{C} \mathrm{NMR}\left(101 \mathrm{MHz}, \mathrm{CDCl}_{3}\right) \delta 184.4,169.6$, 161.6, 150.3, 132.4, 129.9 (2C), 129.7, 128.1 (2C), 127.2 (2C), 125.8, 125.0 (2C), 99.3, 28.1, 14.1. IR, $v_{\max } / \mathrm{cm}^{-1}: 2964,1653$, 1602, 1566, 1439, 1296, 1255, 1183, 1151, 1046. HRMS (ES TOF) 
calcd for $\mathrm{C}_{18} \mathrm{H}_{15} \mathrm{NNaO}_{2}\left([\mathrm{M}+\mathrm{Na}]^{+}\right): 300.0995$, found 300.0997 $(-0.6 \mathrm{ppm})$.

3-(4-Fluorobenzoyl)-5-phenylisoxazole (10da). Product 10da was obtained via the method described for compound 10aa, employing 2-nitro-4'-fluoroacetophenone $\mathbf{1 d}^{27}$ (183 mg, 1.00 $\mathrm{mmol})$ and phenylacetylene $9 \mathrm{a}(102 \mathrm{mg}, 1.00 \mathrm{mmol})$ and purified by column chromatography (eluent benzene/hexane, $1: 2 \mathrm{v} /$ v). White solid, mp $111-113{ }^{\circ} \mathrm{C}(\mathrm{EtOH}), \mathrm{lit}^{32} \mathrm{mp} 112-114{ }^{\circ} \mathrm{C}$; yield $77 \mathrm{mg}(0.29 \mathrm{mmol}, 29 \%) . R_{\mathrm{f}} 0.49$, benzene/hexane $(1: 2, \mathrm{v} /$ v). ${ }^{1} \mathrm{H}$ NMR $\left(400 \mathrm{MHz}, \mathrm{CDCl}_{3}\right) \delta 8.48-8.39(\mathrm{~m}, 2 \mathrm{H}), 7.89-7.82(\mathrm{~m}$, 2H), 7.55-7.48 (m, 3H), 7.25-7.18 (m, 2H), 7.05 (s, 1H). ${ }^{13} \mathrm{C}$ NMR $\left(101 \mathrm{MHz}, \mathrm{CDCl}_{3}\right) \delta 183.0,169.9,165.4(\mathrm{~d}, J=256.8 \mathrm{~Hz}), 161.4$, 132.5 (d, $J=9.5 \mathrm{~Hz}, 2 \mathrm{C}), 131.1$ (d, $J=2.8 \mathrm{~Hz}), 129.8,128.2,125.6$, 125.0, $114.8(\mathrm{~d}, J=21.6 \mathrm{~Hz}, 2 \mathrm{C}), 99.2 . \mathrm{IR}, v_{\max } / \mathrm{cm}^{-1}: 3133,1655$, 1600, 1506, 1441, 1296, 1251, 1231, 1147, 1104. HRMS (ES TOF) calcd for $\mathrm{C}_{16} \mathrm{H}_{10} \mathrm{FNNaO}_{2}\left([\mathrm{M}+\mathrm{Na}]^{+}\right): 290.0588$, found 290.0582 (1.9 ppm).

3-(4-Chlorobenzoyl)-5-phenylisoxazole (10fa). Product 10fa was obtained via the method described for compound 10aa, employing 2-nitro-4'-chloroacetophenone $\mathbf{1}^{27}(199 \mathrm{mg}, 1.00$ $\mathrm{mmol}$ ) and phenylacetylene $9 \mathrm{a}(102 \mathrm{mg}, 1.00 \mathrm{mmol})$ and purified by column chromatography (eluent benzene/hexane, $1: 2 \mathrm{v} /$ v). Yellowish solid, mp $139-141{ }^{\circ} \mathrm{C}(\mathrm{EtOH}), \mathrm{lit}^{32} \mathrm{mp} 143-145{ }^{\circ} \mathrm{C}$; $R_{\mathrm{f}} 0.49$, benzene/hexane $(1: 2, \mathrm{v} / \mathrm{v})$. Yield $105 \mathrm{mg}(0.37 \mathrm{mmol}$, $37 \%) .{ }^{1} \mathrm{H}$ NMR $\left(400 \mathrm{MHz}, \mathrm{CDCl}_{3}\right) \delta 8.38-8.30(\mathrm{~d}, 2 \mathrm{H}), 7.85$ (dd, $J$ $=7.6,2.0 \mathrm{~Hz}, 2 \mathrm{H}), 7.51(\mathrm{~m}, J=8.4,2.0 \mathrm{~Hz}, 5 \mathrm{H}), 7.05(\mathrm{~s}, 1 \mathrm{H}) .{ }^{13} \mathrm{C}$ NMR $\left(101 \mathrm{MHz}, \mathrm{CDCl}_{3}\right) \delta$ 183.4, 169.9, 161.3, 139.7, 133.0, 131.1 (2C), 129.8, 128.2 (2C), 127.9 (2C), 125.6, 125.0 (2C), 99.2. IR, $v_{\max } / \mathrm{cm}^{-1}: 3133,3060,1930,1884,1648,1588,1443,1395,1251$, 1181, 1089. HRMS (ES TOF) calcd for $\mathrm{C}_{16} \mathrm{H}_{10} \mathrm{ClNNaO}_{2}([\mathrm{M}+$ $\mathrm{Na}]^{+}$): 306.0292 , found $306.0295(-0.9 \mathrm{ppm})$.

3-(4-Chlorobenzoyl)-5-pentylisoxazole (10fb). Product $10 \mathrm{fb}$ was obtained via the method described for compound 10aa, employing 2-nitro-4'-chloroacetophenone $\mathbf{1 f}^{\mathbf{2}^{7}}$ (199 mg, 1.00 mmol) and hept-1-yne $9 \mathbf{b}(96 \mathrm{mg}, 1.00 \mathrm{mmol})$ and purified by column chromatography (eluent EtOAc/PE, $1: 4$ ). Colorless oil; $R_{\mathrm{f}} 0.74$, EtOAc/hexane $(1: 4, \mathrm{v} / \mathrm{v})$. Yield $86 \mathrm{mg}(0.31 \mathrm{mmol}, 31 \%)$. ${ }^{1} \mathrm{H}$ NMR (400 MHz, $\left.\mathrm{CDCl}_{3}\right) \delta 8.33-8.28(\mathrm{~m}, 2 \mathrm{H}), 7.53-7.47(\mathrm{~m}$, $2 \mathrm{H}), 6.55(\mathrm{~s}, 1 \mathrm{H}), 2.86(\mathrm{t}, J=7.6 \mathrm{~Hz}, 2 \mathrm{H}), 1.83-1.75(\mathrm{~m}, 2 \mathrm{H}), 1.44-$ $1.35(\mathrm{~m}, 4 \mathrm{H}), 0.95-0.92(\mathrm{~m}, 3 \mathrm{H}) .{ }^{13} \mathrm{C}$ NMR $\left(101 \mathrm{MHz}, \mathrm{CDCl}_{3}\right)$ $\delta$ 184.9, 175.1, 161.9, 140.7, 134.3, 132.2 (2C), 129.0 (2C), 101.8, 31.3, 27.3, 26.7, 22.4, 14.0. IR, $v_{\max } / \mathrm{cm}^{-1}: 2935,2853,1923$, $1665,1590,1455,1400,1255,1210,1178,1096$. HRMS (ES TOF) calcd for $\mathrm{C}_{15} \mathrm{H}_{16} \mathrm{ClNNaO}_{2}\left([\mathrm{M}+\mathrm{Na}]^{+}\right): 300.0762$, found 300.0760 (0.7 ppm).

3-(3-Chlorobenzoyl)-5-phenylisoxazole (10ga). Product 10ga was obtained via the method described for compound 10aa, employing 2-nitro-3'-chloroacetophenone $\mathbf{1 g}^{27}$ (199 mg, 1.00 $\mathrm{mmol})$ and phenylacetylene $9 \mathrm{a}(102 \mathrm{mg}, 1.00 \mathrm{mmol})$ and purified by column chromatography (eluent EtOAc/PE $1: 4$ ). Colorless solid, $\mathrm{mp} 117-117.5{ }^{\circ} \mathrm{C}(\mathrm{EtOH}) ; R_{\mathrm{f}} 0.46$, benzene/hexane $(1: 2, \mathrm{v} / \mathrm{v})$. Yield $99 \mathrm{mg}(0.35 \mathrm{mmol}, 35 \%) .{ }^{1} \mathrm{H}$ NMR $(400 \mathrm{MHz}$, $\left.\mathrm{CDCl}_{3}\right) \delta 8.34(\mathrm{t}, J=2.0 \mathrm{~Hz}, 1 \mathrm{H}), 8.27(\mathrm{~d}, J=7.8 \mathrm{~Hz}, 1 \mathrm{H}), 7.85(\mathrm{~m}$, $J=7.8,2.0 \mathrm{~Hz}, 2 \mathrm{H}), 7.63(\mathrm{~m}, J=8.1,2.2,1.1 \mathrm{~Hz}, 1 \mathrm{H}), 7.54-7.48$ (m, 4H), $7.06(\mathrm{~s}, 1 \mathrm{H}) .{ }^{13} \mathrm{C}$ NMR (101 MHz, $\left.\mathrm{CDCl}_{3}\right) \delta$ 184.5, 171.1, 162.2, 137.2, 134.9, 134.0, 130.9, 130.6, 129.9, 129.2 (2C), 128.9, 126.6, 126.0 (2C), 100.2. IR, $v_{\max } / \mathrm{cm}^{-1}: 1959,1896,1761,1655$,
1571, 1436, 1236, 1159, 1082, 1046. HRMS (ES TOF) calcd for $\mathrm{C}_{16} \mathrm{H}_{10} \mathrm{ClNNaO}_{2}\left([\mathrm{M}+\mathrm{Na}]^{+}\right): 306.0292$, found $306.0285(2.2$ $\mathrm{ppm})$.

5-(Bromomethyl)-3-(4-bromobenzoyl)isoxazole

(10hc). Product 10hc was obtained via the method described for compound 10aa, employing 2-nitro-4'-bromoacetophenone $1 \mathbf{h}^{27}$ $(244 \mathrm{mg}, 1.00 \mathrm{mmol})$ and propargyl bromide $9 \mathrm{c}(119 \mathrm{mg}, 1.00$ mmol) and purified by column chromatography (eluent EtOAc/ PE, $1: 4)$. Brown solid, $\mathrm{mp} 83-85{ }^{\circ} \mathrm{C} ; R_{\mathrm{f}} 0.40$, EtOAc/hexane $(1: 4, \mathrm{v} / \mathrm{v})$. Yield $66 \mathrm{mg}(0.19 \mathrm{mmol}, 19 \%) .{ }^{1} \mathrm{H}$ NMR $(400 \mathrm{MHz}$, $\left.\mathrm{CDCl}_{3}\right) \delta 8.22-8.18(\mathrm{~m}, 2 \mathrm{H}), 7.69-7.66(\mathrm{~m}, 2 \mathrm{H}), 6.85(\mathrm{~d}, J=$ $0.6 \mathrm{~Hz}, 1 \mathrm{H}), 4.54(\mathrm{~s}, 2 \mathrm{H}) .{ }^{13} \mathrm{C}$ NMR $\left(101 \mathrm{MHz}, \mathrm{CDCl}_{3}\right) \delta 184.0$, 168.6, 162.1, 134.1, 132.2 (2C), 132.0 (2C), 129.8, 104.7, 17.9. IR, $v_{\max } / \mathrm{cm}^{-1}: 3147,2920,2853,1934,1652,1585,1448,1397,1295$, 1224, 1178, 932. HRMS (ES TOF) calcd for $\mathrm{C}_{11} \mathrm{H}_{7} \mathrm{Br}_{2} \mathrm{NaNO}_{2}([\mathrm{M}+$ $\left.\mathrm{Na}]^{+}\right): 365.8736$, found 365.8727 (2.4 ppm).

3,4-Dibenzoyl-5-phenyl-4,5-dihydroisoxazole (12aa) and 3,5dibenzoyl-4-phenyl-4,5-dihydroisoxazole (14aa). 2-Nitroacetophenone $\mathbf{1 a}^{27}(165 \mathrm{mg}, 1.00 \mathrm{mmol})$ and trans-chalcone 11a (312 mg, $1.5 \mathrm{mmol}$ ) were combined with to $2.0 \mathrm{~g}$ of polyphosphoric acid $\left(80 \mathrm{wt} \%\right.$ of $\left.\mathrm{P}_{2} \mathrm{O}_{5}\right)$. The reaction mixture was stirred at $130{ }^{\circ} \mathrm{C}$ for $3-5 \mathrm{~h}$ (TLC control). Reaction mixture was diluted with water and extracted with ethyl acetate $(4 \times 15 \mathrm{~mL})$. Combined organic layers were washed with brine, and concentrated in vacuo. The residue was purified by column chromatography on silica gel (eluent: EtOAc/PE, $1: 4 \mathrm{v} / \mathrm{v}$ ) to obtain two regioisomeric products 12aa and 14aa.

Product 12aa. Brown solid, mp $105-107{ }^{\circ} \mathrm{C} ; R_{\mathrm{f}} 0.30$, EtOAc/ hexane $(1: 4, \mathrm{v} / \mathrm{v})$. Yield $138 \mathrm{mg}(0.39 \mathrm{mmol}, 39 \%) .{ }^{1} \mathrm{H}$ NMR $\left(400 \mathrm{MHz}, \mathrm{CDCl}_{3}\right) \delta 8.27(\mathrm{dt}, J=8.3,1.3 \mathrm{~Hz}, 2 \mathrm{H}), 7.90-7.85(\mathrm{~m}$, 2H), 7.62 (dddd, $J=7.5,5.2,3.9,1.9 \mathrm{~Hz}, 2 \mathrm{H}), 7.52-7.41(\mathrm{~m}, 7 \mathrm{H})$, $7.33(\mathrm{dd}, J=6.3,2.6 \mathrm{~Hz}, 2 \mathrm{H}), 5.78(\mathrm{~d}, J=8.2 \mathrm{~Hz}, 1 \mathrm{H}), 5.58(\mathrm{dd}, J$ $=8.3,1.1 \mathrm{~Hz}, 1 \mathrm{H}) .{ }^{13} \mathrm{C} \mathrm{NMR}\left(101 \mathrm{MHz}, \mathrm{CDCl}_{3}\right) \delta 195.7,185.8$, 157.1, 138.3, 135.6, 135.5, 134.4, 134.1, 130.7 (2C), 129.5, 129.4 (2C), 129.3 (2C), 129.1 (2C), 128.6 (2C), 126.4 (2C), 89.5, 63.1. IR, $v_{\max } / \mathrm{cm}^{-1}: 3060,2925,1684,1643,1578,1448,1366,1277,1195$, 1016, 932. HRMS (ES TOF) calcd for $\mathrm{C}_{23} \mathrm{H}_{17} \mathrm{NaNO}_{3}\left([\mathrm{M}+\mathrm{Na}]^{+}\right)$: 378.1101, found 378.1091 (2.6 ppm).

Product 14aa. Yellow viscous oil; $R_{\mathrm{f}} 0.29$, EtOAc/hexane $(1: 4$, $\mathrm{v} / \mathrm{v})$. Yield $121 \mathrm{mg}(0.34 \mathrm{mmol}, 34 \%) .{ }^{1} \mathrm{H}$ NMR $\left(400 \mathrm{MHz}, \mathrm{CDCl}_{3}\right)$ $\delta 8.17(\mathrm{dt}, J=8.5,1.2 \mathrm{~Hz}, 2 \mathrm{H}), 8.01(\mathrm{dt}, J=8.5,1.2 \mathrm{~Hz}, 2 \mathrm{H}), 7.66-$ $7.56(\mathrm{~m}, 2 \mathrm{H}), 7.53-7.32(\mathrm{~m}, 9 \mathrm{H}), 5.86(\mathrm{dd}, J=5.4,1.0 \mathrm{~Hz}, 1 \mathrm{H})$, $5.37(\mathrm{dd}, J=5.4,1.0 \mathrm{~Hz}, 1 \mathrm{H}) .{ }^{13} \mathrm{C}$ NMR $\left(101 \mathrm{MHz}, \mathrm{CDCl}_{3}\right)$ $\delta$ 192.1, 184.9, 159.1, 137.6, 135.9, 134.4, 134.0, 133.8, 130.5 (2C), 129.6 (2C), 129.6 (2C), 129.1 (2C), 128.5 (2C), 128.47, 127.8 (2C), 90.6, 55.4. IR, $v_{\max } / \mathrm{cm}^{-1}: 3062,2927,1686,1645,1579$, 1448, 1369, 1279, 1196, 1026, 932. HRMS (ES TOF) calcd for $\mathrm{C}_{23} \mathrm{H}_{17} \mathrm{NaNO}_{3}\left([\mathrm{M}+\mathrm{Na}]^{+}\right): 378.1101$, found 378.1092 (2.3 ppm).

3-(4-Chlorobenzoyl)-4-(2-nitrobenzoyl)-5-(2-chlorophenyl) isoxazole (15fb). Product 15gb was obtained via the method described for compound 12aa, employing 2-nitro-4'-chloroacetophenone 1f (199 mg, $1.00 \mathrm{mmol})$, and (E)-2-chloro-2'nitrochalcone $\mathbf{1 1 b}^{25}$ (431 mg, $1.5 \mathrm{mmol}$ ) and purified by column chromatography (eluent EtOAc/PE, $1: 4$ ). Brown viscous oil; $R_{\mathrm{f}}$ $=0.41$, benzene. Yield $248 \mathrm{mg}(0.53 \mathrm{mmol}, 53 \%) .{ }^{1} \mathrm{H}$ NMR $(400$ $\left.\mathrm{MHz}, \mathrm{CDCl}_{3}\right) \delta 8.05-7.92(\mathrm{~m}, 2 \mathrm{H}), 7.77(\mathrm{dd}, J=8.5,1.2 \mathrm{~Hz}, 1 \mathrm{H})$, 7.56-7.49 (m, 3H), 7.41-7.16 (m, 6H). ${ }^{13} \mathrm{C}$ NMR (101 MHz, 
$\left.\mathrm{CDCl}_{3}\right) \delta 185.0,184.3,171.0,160.2,145.8,141.6,134.3,134.1$, 133.9, 133.9, 132.7, 131.5 (2C), 131.4, 131.3, 129.9, 129.4 (2C), 129.1, 126.8, 125.0, 123.9, 118.6. IR, $v_{\max } / \mathrm{cm}^{-1}$ : 3089, 2925, 1732, 1674, 1587, 1522, 1346, 1224, 1084, 1014, 930. HRMS (ES TOF) calcd for $\mathrm{C}_{23} \mathrm{H}_{12} \mathrm{Cl}_{2} \mathrm{~N}_{2} \mathrm{NaO}_{5}\left([\mathrm{M}+\mathrm{Na}]^{+}\right)$: 489.0015 , found 489.0013 (0.6 ppm).

3-Benzoyl-5-phenyl-3a,6a-dihydro-4H-pyrrolo[3,4- $d]$ iso-

xazole-4,6(5H)-dione (17a). Product 17a was obtained via the method described for compound 10aa, employing 2-nitroacetophenone $\mathbf{1 a}^{27}$ (165 mg, $\left.1.00 \mathrm{mmol}\right)$ and $N$-phenylmaleimide 16 (173 mg, $1.00 \mathrm{mmol}$ ) and purified by column chromatography (eluent EtOAc/PE $1: 4$ ). Colorless solid, $\mathrm{mp}$ 147-150 ${ }^{\circ} \mathrm{C}$, lit ${ }^{33} \mathrm{mp} 100-102{ }^{\circ} \mathrm{C}$ (EtOAc); $R_{\mathrm{f}} 0.16$, EtOAc/hexane (1 : 4, v/v). Yield $201 \mathrm{mg}(0.63 \mathrm{mmol}, 63 \%) .{ }^{1} \mathrm{H}$ NMR $(400 \mathrm{MHz}$, $\left.\mathrm{CDCl}_{3}\right) \delta$ 8.27-8.17 (m, 2H), 7.68-7.62 (m, 1H), 7.54-7.42 (m, $5 \mathrm{H}), 7.31-7.26(\mathrm{~m}, 2 \mathrm{H}), 5.70(\mathrm{~d}, J=9.8 \mathrm{~Hz}, 1 \mathrm{H}), 5.24(\mathrm{~d}, J=$ $9.8 \mathrm{~Hz}, 1 \mathrm{H}) .{ }^{13} \mathrm{C}$ NMR (101 MHz, $\left.\mathrm{CDCl}_{3}\right) \delta 183.4,170.0,168.8$, 153.2, 134.9, 134.7, 130.8, 130.6 (2C), 129.5 (2C), 129.4, 128.8 (2C), 126.2 (2C), 81.2, 54.9. IR, $v_{\max } / \mathrm{cm}^{-1}: 3065,1901,1713$, 1651, 1598, 1501, 1448, 1381, 1279, 1200, 1070, 1024. HRMS (ES TOF) calcd for $\mathrm{C}_{18} \mathrm{H}_{12} \mathrm{~N}_{2} \mathrm{NaO}_{4}\left([\mathrm{M}+\mathrm{Na}]^{+}\right)$: 343.0689, found 343.0679 (2.9 ppm).

\section{Author contributions}

A. V. Aksenov - conceptualization, supervision, funding acquisition; N. A. Aksenov - methodology, formal analysis, funding acquisition; N. K. Kirilov - investigation; A. A. Skomorokhov - investigation; D. A. Aksenov - investigation; I. A. Kurenkov - investigation; E. A. Sorokina - investigation, formal analysis; M. A. Nobi - formal analysis, writing (review and editing); M. Rubin - conceptualization, supervision, writing (original draft, review, and editing).

\section{Conflicts of interest}

There are no conflicts to declare.

\section{Acknowledgements}

This work was supported by the grant of the President of the Russian Federation (grant \#MD-3505.2021.1.3) and the Ministry of Education and Science of the Russian Federation (grant \#0795-2020-0031). Support for the NMR instruments used in this project was provided by the Center of Shared Instrumentation, NCFU (Grant \#075-15-2021-672).

\section{Notes and references}

1 R. Ballini, L. Barboni, L. Castrica, F. Fringuelli, D. Lanari, F. Pizzo and L. Vaccaro, Adv. Synth. Catal., 2008, 350, 12181224.

2 E. M. Budynina, K. L. Ivanov, A. O. Chagarovskiy, V. B. Rybakov, I. V. Trushkov and M. Y. Melnikov, Chem.Eur. J., 2016, 22, 3692-3696.

3 M. N. Grayson, J. Org. Chem., 2017, 82, 4396-4401.
4 Y. Liu, W. Zhen, W. Dai, F. Wang and X. Li, Org. Lett., 2013, 15, 874-877.

5 G. R. Reddy, D. Mukherjee, A. K. Chittoory and S. Rajaram, Org. Lett., 2014, 16, 5874-5877.

6 D. K. Romney, N. S. Sarai and F. H. Arnold, ACS Catal., 2019, 9, 8726-8730.

7 H. Ueda, K. Yoshida and H. Tokuyama, Org. Lett., 2014, 16, 4194-4197.

8 L. Wen, L. Yin, Q. Shen and L. Lu, ACS Catal., 2013, 3, 502506.

9 R. Ballini and M. Petrini, Tetrahedron, 2004, 60, 1017-1047.

10 R. Ballini and M. Petrini, Adv. Synth. Catal., 2015, 357, 23712402.

11 R. Ballini, M. Petrini and G. Rosini, Molecules, 2008, 13, 319330.

12 R. Ballini, M. Petrini and G. Rosini, Molecules, 2008, 13, 319330.

13 A. V. Aksenov, V. Khamraev, N. A. Aksenov, N. K. Kirilov, D. A. Domenyuk, V. A. Zelensky and M. Rubin, RSC Adv., 2019, 9, 6636-6642.

14 D. A. Aksenov, N. A. Arutiunov, V. V. Maliuga, A. V. Aksenov and M. Rubin, Beilstein J. Org. Chem., 2020, 16, 2903-2910.

15 N. A. Aksenov, A. V. Aksenov, N. K. Kirilov, N. A. Arutiunov, D. A. Aksenov, V. Maslivetc, Z. Zhao, L. Du, M. Rubin and A. Kornienko, Org. Biomol. Chem., 2020, 18, 6651-6664.

16 N. A. Aksenov, A. V. Aksenov, S. N. Ovcharov, D. A. Aksenov and M. Rubin, Front. Chem., 2020, 8, 77.

17 N. A. Aksenov, N. A. Arutiunov, N. K. Kirillov, D. A. Aksenov, A. V. Aksenov and M. Rubin, Chem. Heterocycl. Compd., 2020, 56, 1067-1072.

18 A. V. Aksenov, N. A. Aksenov, O. N. Nadein and I. V. Aksenova, Synlett, 2010, 2628-2630, DOI: 10.1055/s0030-1258767.

19 A. V. Aksenov, N. A. Aksenov, O. N. Nadein and I. V. Aksenova, Synth. Commun., 2012, 42, 541-547.

20 A. V. Aksenov, A. N. Smirnov, N. A. Aksenov, A. S. Bijieva, I. V. Aksenova and M. Rubin, Org. Biomol. Chem., 2015, 13, 4289-4295.

21 N. A. Aksenov, A. V. Aksenov, O. N. Nadein, D. A. Aksenov, A. N. Smirnov and M. Rubin, RSC Adv. , 2015, 5, 71620-71626.

22 K.-i. Itoh, T. Aoyama, H. Satoh, Y. Fujii, H. Sakamaki, T. Takido and M. Kodomari, Tetrahedron Lett., 2011, 52, 6892-6895.

23 A. V. Aksenov, A. N. Smirnov, I. V. Magedov, M. R. Reisenauer, N. A. Aksenov, I. V. Aksenova, A. L. Pendleton, G. Nguyen, R. K. Johnston, M. Rubin, A. De Carvalho, R. Kiss, V. Mathieu, F. Lefranc, J. Correa, D. A. Cavazos, A. J. Brenner, B. A. Bryan, S. Rogelj, A. Kornienko and L. V. Frolova, J. Med. Chem., 2015, 58, 2206-2220.

24 M. S. Maurice and S. L. Bearne, Biochemistry, 2004, 43, 25242532.

25 N. A. Aksenov, D. A. Aksenov, A. A. Skomorokhov, L. A. Prityko, A. V. Aksenov, G. D. Griaznov and M. Rubin, J. Org. Chem., 2020, 85, 12128-12146.

26 Z. Wang, X. Wu, Z. Li, Z. Huang and F. Chen, Org. Biomol. Chem., 2019, 17, 3575-3580. 
27 Z. Lian, S. D. Friis and T. Skrydstrup, Chem. Commun., 2015, 51, 3600-3603.

28 Q. Song, Q. Feng and K. Yang, Org. Lett., 2014, 16, 624-627.

29 A. R. Daniewski, M. Witanowski and T. Urbanski, J. Org. Chem., 1967, 32, 4050-4052.

30 H. R. Snyder and N. E. Boyer, J. Am. Chem. Soc., 1955, 77, 4233-4238.
31 L. Tang, X. Guo, Y. Li, S. Zhang, Z. Zha and Z. Wang, Chem. Commun., 2013, 49, 5213-5215.

32 P. Gao, H.-X. Li, X.-H. Hao, D.-P. Jin, D.-Q. Chen, X.-B. Yan, X.-X. Wu, X.-R. Song, X.-Y. Liu and Y.-M. Liang, Org. Lett., 2014, 16, 6298-6301.

33 H. Wang, R. Cheng, G. Wang, Y. Shi, J. Wang, H. Guo, L. Trigoura, Y. Xing and S. Sun, Tetrahedron Lett., 2020, 61, 151652 . 